Hautarzt 2018 $69: 783-784$

https://doi.org/10.1007/s00105-018-4260-0

Online publiziert: 24. August 2018

(c) Springer Medizin Verlag GmbH, ein Teil von Springer Nature 2018

CrossMark

\title{
M. Meurer
}

Stiftung Hochschulmedizin Dresden, Universitätsklinikum und Medizinische Fakultät Carl Gustav Carus, Technische Universität Dresden, Dresden, Deutschland

\section{Nachruf auf Herrn Prof. Dr. med. Dr. h.c. mult. Otto Braun-Falco}

Mühen des hohen Alters wohl erleichterten.

Am 23. April 2018 traf sich in der Harlachinger Pfarrkirche Maria Immaculata die Familie des Verstorbenen inmitten eines großen Kreises an persönlichen Freunden, akademischen Schülern, Vertretern der Medizinischen Fakultät der Ludwig-Maximilians-Universität und ehemaligen Mitarbeitern - darunter die unverändert liebenswerte Schwester Coelesta - der durch seinen Vorgänger Prof. Dr. Alfred Marchionini und ihn groß und international berühmt gewordenen Klinik und Poliklinik der LMU München, die Prof. Braun-Falco von 1967 bis 1991 geleitet hatte, um von ihm ehrenden Abschied zu nehmen.

Die öffentliche Anerkennung des Trägers des Großen Verdienstkreuzes des Verdienstordens der Bundesrepublik Deutschland, des Bayerischen Maximiliansordens für Wissenschaft und Kunst, des Bayerischen Verdienstordens, der Bayerischen Staatsmedaille für soziale Verdienste, der Medaille „München leuchtet“ in Gold der Landeshauptstadt, des „Order of the Rising Sun“ der Japanischen Staatsregierung sowie zahlreicher weiterer hoher nationaler und internationaler Auszeichnungen wurde dabei ebenso gewürdigt wie - in der anrührend persönlichen Rede seines Sohnes Markus - die private Sphäre des Verstorbenen als Ehemann der unvergessenen Sissy Braun-Falco, als treusorgender Vater und beratender Mentor sowie als stolzer Großvater in Erinnerung gerufen.

Prof. Dr. Enno Christopher verstand es in seiner Abschiedsrede, die Besonderheit des großen akademischen Lehrers, Wissenschaftlers und Arztes Otto Braun-Falco gegenwärtig werden zu las- sen: Seine menschliche Zuwendung und Disziplin in der ärztlichen Tätigkeit, seine große Gabe, als Hochschullehrer, Klinikleiter und Autor Wissen und Erfahrung begeisternd zu vermitteln, seine Neugier, Inspiration und visionäre Vorausschau als Wissenschaftler und schließlich seine angeborene Befähigung zur Kommunikation und Führung haben ihn, meist in kollegialer Abstimmung mit Freunden und Mitstreitern, zum souveränen Lenker der deutschen und internationalen Dermatologie seiner Ära werden lassen und seine Schüler weit über die gemeinsame Münchner Zeit hinaus geprägt.

Im Rahmen der anschließenden Aussegnung auf dem nahe liegenden Waldfriedhof Grünwald wurde dem Verstorbenen von Prof. Dr. Thomas Ruzicka im Namen aller derzeitigen Mitarbeiter der Münchner Universitäts-Hautklinik mit ehrenden und herzlichen Worten gedankt, Prof. Dr. Tilo Biedermann ging in seiner Rede als Vertreter der Deutschen Dermatologischen Gesellschaft auf die entscheidenden Verdienste von Prof. Braun-Falco um die inhaltliche Ausweitung unseres Fachgebietes und seine Verknüpfung mit benachbarten Disziplinen sowie auf dessen fortbestehende Vorbildfunktion für nachrückende Jahrgänge von deutschen Dermatologen ein.

Prof. Dr. Otto Braun-Falco war dem Springer-Verlag und unserer Zeitschrift Der Hautarzt als Doyen der Deutschen Dermatologie mehr als 50 Jahre verbunden:Erhat 6 Auflagen „seines“Lehrbuchs im Springer-Verlag als Herausgeber, Mitherausgeber bzw. Namensgeber verantwortet. Der „Braun-Falco“ wurde das Referenzwerk der Dermatologie für Generationen deutschsprachiger und später auch englischsprachiger Studenten und 
Hautärzte und zum ständigen Begleiter im Studien- oder Berufsalltag.

In dem 2012 publizierten und immer noch lesenswerten Sonderheft von Der Hautarzt als Festschrift zum 90. Geburtstag [1] wurden die z.T. epochalen Verdienste von Prof. Dr. Otto Braun-Falco für unser Fach dokumentiert. Beispielhaft genannt seien die Einführung der Lasertherapie und neuer diagnostischer Verfahren wie Elektronenmikroskopie und Immunhistologie in unser Fachgebiet, die Förderung der Dermatochirurgie, dermatologischen Onkologie und Allergologie sowie die frühzeitige Einbindung der Dermatologie in die Betreuung von Patienten mit HIV-Infektion.

Die Festschrift dokumentiert auch die historische Weitsicht, mit der Prof. Dr. Braun-Falco - unter anderen politischen Rahmenbedingungen als heute - die multilateralen Beziehungen der deutschen Dermatologie mit europäischen, v.a. osteuropäischen Nachbarn und internationalen Freunden ausgebaut und damit das Lebenswerk seines Vorgängers Alfred Marchionini fortgesetzt und intensiviert hat. Viele dieser Verbindungen sind noch aktiv und werden weltweit von Dermatologinnen und Dermatologen getragen, die den Dank und das Gedenken an Otto Braun-Falco, so wie wir es tun, lebendig halten.

Dresden, im Juli 2018

Prof. Dr. Michael Meurer im Namen der Schriftleitung, aller Herausgeber und des nationalen sowie internationalen Beirates der Zeitschrift Der Hautarzt

\section{Korrespondenzadresse}

\section{Prof. Dr. M. Meurer}

Stiftung Hochschulmedizin Dresden, Universitätsklinikum und Medizinische Fakultät Carl Gustav Carus, Technische Universität Dresden

Fetscherstr. 74, 01307 Dresden, Deutschland michael.meurer@uniklinikum-dresden.de

\section{Literatur}

1. Hautarzt 2012.63 (Suppl 1): 5-76. Sonderheft zu Ehren des Verstorbenen

\section{P. Härle}

\section{Rheumalabor 2018/2019}

Aus der Praxis, für die Praxis

Berlin: epubli 2018, 1. Auflage, 124 S., (ISBN: 978-3746734392), 32,00 EUR

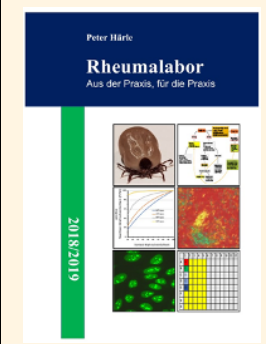

Nach wie vor sind die labormedizinischen Untersuchungen ein essenzieller Bestandteil der Diagnose und Differentialdiagnostik systemisch

entzündlicher Erkrankungen, und somit des gesamten Spektrums der Entitäten des rheumatologischen und klinischimmunologischen Formenkreises.

Aus diesen Gründen sind die Indikation, die technische Durchführung und die Interpretation der klinisch-immunologischen Labormethoden auch zwingender Bestandteil des Weiterbildungskataloges - nicht nur für den internistisch tätigen Rheumatologen. Auf der anderen Seite bedingt das schmale Budget des Rheumatologen einen überlegten und zielgerichteten Einsatz aller Labortests, um möglichst effizient die Diagnostik gestalten zu können. Ein „zu viel messen“ führt daher häufig zu Interpretationsschwierigkeiten, vor allem bei grenzwertigen Befunden, die mit der eigentlichen Grunderkrankung nur wenig zu tun haben.

Herr Prof. Peter Härle hat sich diesem alltäglichen Problem in einer sehr praxisorientierten Weise genähert, in dem er zusammen mit der Erfahrung von langjährigen Wegbegleitern ein handliches Buch zu dieser Thematik zusammengestellt hat.

Ausgehend vom geschichtlichen Hintergrund von Laborbestimmungen, über die Erklärung der derzeit gängigen Labortechniken, hin zu den allermeisten für den klinischen Alltag in der Rheumatologie benötigten Laborbestimmungen und deren Interpretation, beinhaltet es die notwendigen Informationen die es für eine handliche Hilfestellung im Alltag benötigt. Dies wird zusätzlich ergänzt durch zahlreiche Abbildungen, die das Buch trotz des immensen enthaltenen Wissens gut und anschaulich lesbar machen. Auch neueste Erkenntnisse, vor allem in der Differenzialdiagnostik von neuen Antikörpern, sind bereits enthalten. Einziger Wermutstropfen sind hierbei noch die noch etwas zu häufig vorhandenen Formatierungs- und Schreibfehler.

Trotz des all gegenwärtig verfügbaren Internets kann das Buch uneingeschränkt jedem, der rheumatologische Patienten betreut, empfohlen werden, da als "Alleinstellungsmerkmal" die Information schnell verfügbar und direkt am Patienten anwendbar ist.

Da seitens Herrn Kollegen Härle geplant ist, das Buch regelmäßig auf den neuesten Stand zu bringen, ist ein konstantes Einbringen neuester Informationen bereits vorgesehen. Herr Kollege Härle ist auch für jede Verbesserung und neue wissenschaftliche oder klinische Erkenntnisse, die ihm zugetragen werden, dankbar, um so sein Buch mit Hilfe des gesammelten rheumatologischen Kollektivwissens auf dem aktuellsten Stand zu halten. Sicher wird auch der ein oder andere Kollege bei bestimmten Punkten eine etwas andere Meinung haben, aber auch dies soll die Diskussion eher befruchten als den Wert des schönen Werkes schmälern.

Herzlichen Glückwunsch zu diesem gelungenen Buch, dem viel Erfolg beschieden sein soll.

U. Müller-Ladner (Bad Nauheim) 